\title{
A non-tree-based comprehensive study of metazoan Hox and ParaHox genes prompts new insights into their origin and evolution
}

\author{
Morgane Thomas-Chollier ${ }^{1,2,6^{*}}$, Valérie Ledent ${ }^{3}$, Luc Leyns ${ }^{2}$, Michel Vervoort ${ }^{4,5}$
}

\begin{abstract}
Background: Hox and the closely-related ParaHox genes, which emerged prior to the divergence between cnidarians and bilaterians, are the most well-known members of the ancient genetic toolkit that controls embryonic development across all metazoans. Fundamental questions relative to their origin and evolutionary relationships remain however unresolved. We investigate here the evolution of metazoan Hox and ParaHox genes using the HoxPred program that allows the identification of Hox genes without the need of phylogenetic tree reconstructions.
\end{abstract}

Results: We show that HoxPred provides an efficient and accurate classification of Hox and ParaHox genes in their respective homology groups, including Hox paralogous groups (PGs). We analyzed more than 10,000 sequences from 310 metazoan species, from 6 genome projects and the complete UniProtKB database. The HoxPred program and all results arranged in the Datab'Hox database are freely available at http://cege.vub.ac.be/hoxpred/. Results for the genome-scale studies are coherent with previous studies, and also brings knowledge on the Hox repertoire and clusters for newly-sequenced species. The unprecedented scale of this study and the use of a non-tree-based approach allows unresolved key questions about Hox and ParaHox genes evolution to be addressed.

Conclusions: Our analysis suggests that the presence of a single type of Posterior Hox genes (PG9-like) is ancestral to bilaterians, and that new Posterior PGs would have arisen in deuterostomes through independent gene duplications. Four types of Central genes would also be ancestral to bilaterians, with two of them, PG6- and PG7like that gave rise, in protostomes, to the UbdA- and ftz/Antp/Lox5-type genes, respectively. A fifth type of Central genes (PG8) would have emerged in the vertebrate lineage. Our results also suggest the presence of Anterior (PG1 and PG3), Central and Posterior Hox genes in the cnidarians, supporting an ancestral four-gene Hox cluster. In addition, our data support the relationship of the bilaterian ParaHox genes Gsx and Xlox with PG3, and Cdx with the Central genes. Our study therefore indicates three possible models for the origin of Hox and ParaHox in early metazoans, a two-gene (Anterior/PG3 - Central/Posterior), a three-gene (Anterior/PG1, Anterior/PG3 and Central/ Posterior), or a four-gene (Anterior/PG1 - Anterior/PG3 - Central - Posterior) ProtoHox cluster.

\section{Background}

Hox genes encode a large family of closely-related transcription factors from the homeobox class that is characterised by a 60 amino acids region called the homeodomain $[1,2]$. These genes play crucial roles in the development of metazoans, principally by controlling the patterning along the anteroposterior axis in a wide

\footnotetext{
* Correspondence: morgane@bigre.ulb.ac.be

'Laboratoire de Bioinformatique des Génomes et des Réseaux (BiGRe), Université Libre de Bruxelles, Campus Plaine, CP 263, Boulevard du Triomphe, B-1050 Bruxelles, Belgium
}

variety of animals (e.g. $[3,4])$. Their role in the tetrapod limb differentiation is also well-known (reviewed in [5]). Hox genes are usually organized in clusters whose genomic organization reflects domains of expression along the anteroposterior axis (spatial colinearity) [6], as well as, in some species, timing of expression during development (temporal colinearity) [7,8]. Members of this gene family have been reported in both bilaterians (animals presenting a bilateral symmetry) and cnidarians (group including sea anemones, corals, jellyfish), which 
suggests that Hox genes emerged prior to the divergence between bilaterians and cnidarians [9-12].

The ParaHox genes, Gsx (genomic screened homeobox), Xlox (Xenopus laevis homeobox 8) and Cdx (Caudal-type homeobox), are closely related to the Hox genes, and are also involved in developmental processes. Like the Hox genes, they encompass a homeodomain region and form a cluster, at least in chordates (the individual genes are present in non chordate species but are usually scattered in the genome) [13-16]. It is widely believed that the presence of a cluster of three ParaHox genes, although observed so far only in chordates, is ancestral to bilaterians [11,13-15,17-19].

Hox and ParaHox genes have been classified in homology groups, which serve as basis to study their evolutionary relationships $[14,20,21]$ and infer their origin in early metazoans [9-12]. In vertebrates, Hox genes are classified in 14 Paralogous Groups (PGs) [22] that can themselves be grouped in broader classes, known as Anterior (PG1-3), Central (PG4-8) and Posterior (PG914) (e.g. [23]). In some studies, the PG3 proteins have been proposed to form a 4 th independent class (e.g. [14]), although their homeodomain shows a high similarity with that of PG2 proteins [18,24] (Additional file 1, Figure S1). Hox genes from non-vertebrate bilaterian species have been assigned to the aforementioned classes, suggesting that these classes represent ancient types of Hox genes. The ParaHox genes form three groups, named Gsx, Xlox and Cdx [13], yet, they are more closely related to some Hox PGs than to each other. Gs $x$ genes have been reported to be closer to Hox Anterior group genes, $X l o x$ to PG3 genes, and $C d x$ to Hox Posterior genes [13,25-27]. This has lead to the model that the Hox and ParaHox clusters arose through the duplication of a hypothetical ProtoHox cluster (reviewed in [18]).

Most studies aiming at understanding Hox and ParaHox gene evolution used phylogenetic tree reconstruction based on multiple alignments of their homeodomain. Such trees often lack resolution [28], thereby preventing a clear assignment of sequences in homology groups (e.g. [12,29]). Nodes of the trees frequently have low statistical support values that can be explained by the short length of the homeodomain $(60$ amino acids) and its strong conservation [23,30]. Different tree reconstruction methods may furthermore produce conflicting results, giving rise to controversial conclusions $[12,27,31,32]$. Complementary methods, such as sequence similarity, position of the genes in the cluster and Hox/ParaHox signatures [33,34], thus may provide crucial information about the evolution of these genes.

HoxPred [35,36] is a Hox-dedicated program designed to classify Hox protein sequences, without phylogenetic reconstructions. Figure 1 illustrates the general approach; see [35] for a full description of the method. The underlying principle is an extension of the Hox signatures. However, instead of attempting to explicitly discover the few key positions that would define a given homology group, the homeodomain is considered in its entirety as a motif, and described as a generalised profile (Figure 1A). Optimal combinations of such profiles allow the classification of sequences, through a supervised classification approach (Figure 1B) in which discriminant functions are trained to assign sequences to predefined homology groups (Figure 1C). This technique thus differs from pattern search techniques (as used in [37] for homeobox sequences) where a sequence either matches or not a given pattern that describes qualitatively a motif. The profiles used here are quantitative motif descriptors that are more flexible [38], and that take into account amino acid substitutability for all positions of the motif in a residue-specific way i.e. substitution $G$ to $A$ is less penalized than $G$ to $W$. The discriminant functions of HoxPred moreover allows to use the information of multiple profiles, which increases the accuracy of the predictions [35]. These discriminant functions finally return posterior probabilities for all possible homology groups, thereby providing confidence estimates for the predictions [35] (Figure 1B). HoxPred was originally designed for vertebrate sequences classification, and has already proven successful in clarifying the evolutionary history of the HoxC1a genes in teleost fish [39].

Although the origin and evolution of the Hox and ParaHox genes have been addressed by a huge number of studies over this last decade, several fundamental and unsolved questions remain $[10,14,21,29,40,41]$. How did this family emerge in early metazoans? What are the evolutionary relationships between Hox and ParaHox genes? How do cnidarian genes relate to the different classes of bilaterian Hox and ParaHox genes? How did Central and Posterior genes evolve among bilaterians?

We wondered whether a non-tree-based approach could shed light on these crucial questions. In this study, we re-analyzed all published sequence datasets and several newly-sequenced genomes using new versions of the HoxPred program. The extensive evaluation of HoxPred predictions confirmed its accuracy on bilaterian sequences. Its computational efficiency allowed us to simultaneously investigate 310 metazoan species accounting for more than 10,000 homeodomain genes, and infer evolutionary scenarios for the emergence of homologous groups. We found that among the Central Hox genes, PG4, PG5, PG6 and PG7 were likely present in the last common ancestor of bilaterians (Urbilateria). PG8 emerged in vertebrates. For the Posterior Hox genes, PG9 would have been present in Urbilateria; new 


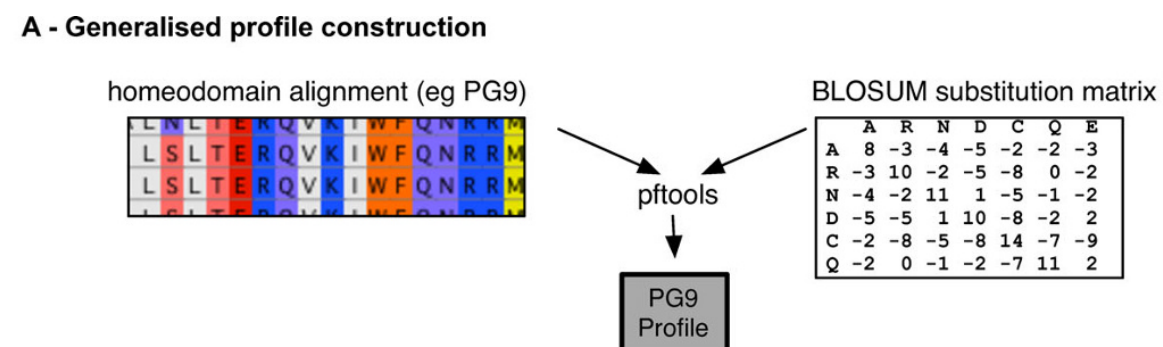

B - HoxPred classification principle

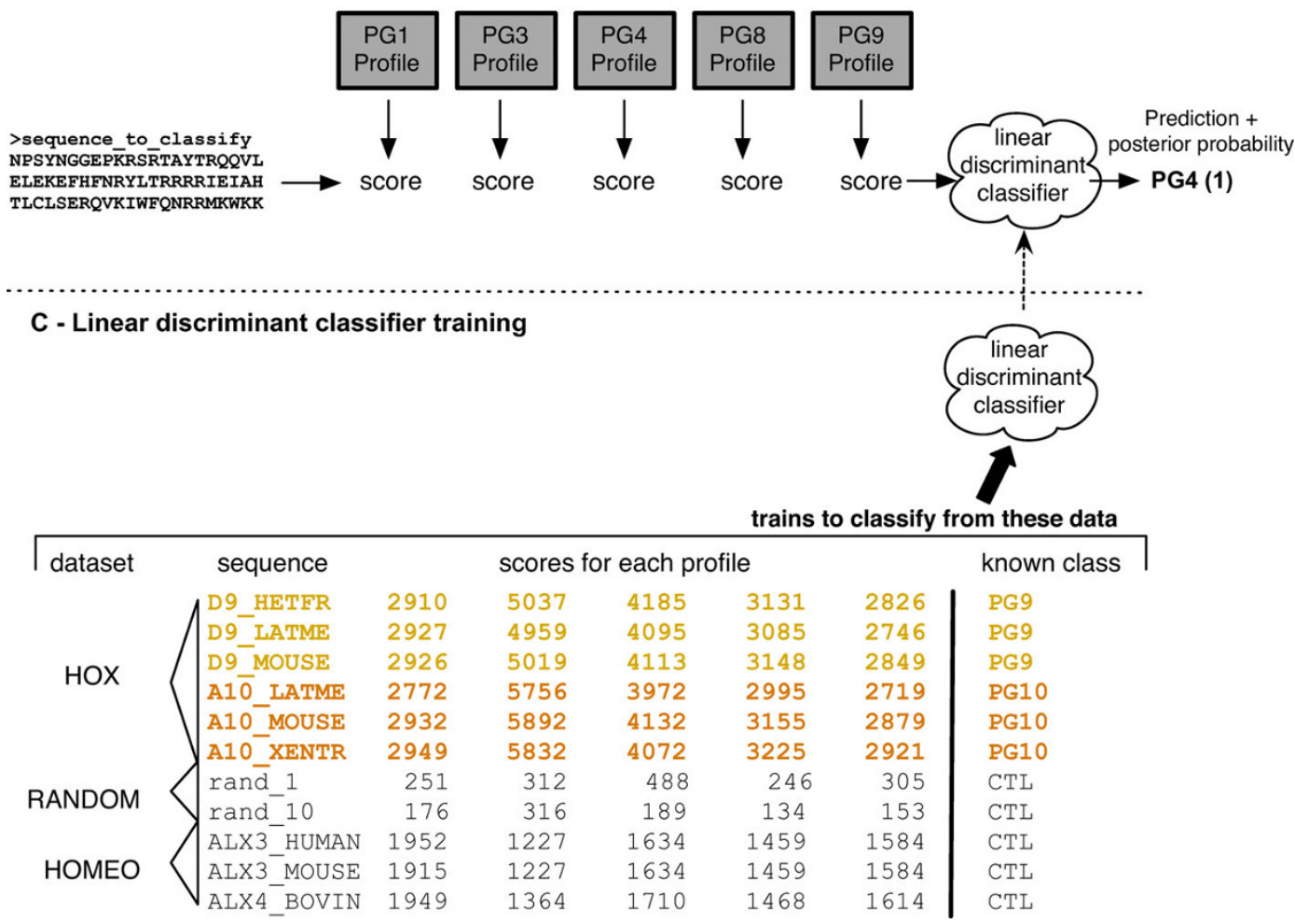

Figure 1 HoxPred classification approach. A. Generalised profile construction. A multiple alignment is built from a set of non-redundant homeodomain sequences that belong to a given homology group (PG9 for this illustration). This alignment then serves as input to a program from the pftools suite [62], which generates the corresponding generalised profile. This profile is a scoring matrix that allows to assign a score to a sequence, based on its similarity with the profile. Contrary to more simple pattern search technique, a profile can provide scores for residues that were not originally found at a given position of the motif. These scores are residue-specific, and extrapolated by using a substitution matrix when building the profile. B. HoxPred classification principle. The sequence to classify is scored by an optimal combination of profiles. The resulting vector of scores then serves as input to a discriminant function that has been previously trained to classify such a vector of scores into a specific class (eg PG4). C. Linear discriminant classifier training. The training phase aims at generating the discriminant function. The training dataset comprise sequences for which the class is known. They can be HOX, RANDOM or HOMEO sequences (see Materials and methods). All sequences are scored by the profiles, so that each sequence is represented by a vector of scores. The classifier is then trained to classify such vector of scores into their associated class (specified on the right). CTL is the control class (see Materials and methods).

paralogous groups then emerged in deuterostomes (group including echinoderms and chordates). PG14 appears in vertebrates, suggesting that the amphioxus Hox14 gene is not related to this PG14. Altogether, our results favor the independent duplication model over the 'Deuterostome Posterior Flexibility' model alone, for the evolution of the Posterior Hox genes. Regarding cnidarians, our results clearly indicate the presence of
Central Hox genes, an observation which contradicts commonly-accepted views [12,27]. Our analysis of ParaHox genes indicates that Gsx is related to PG3 rather than to PG1, across all metazoans. The ParaHox $C d x$ gene would be closer to Central than to Posterior genes. The Xlox genes from bilaterians appear mostly related to PG3, while the few known cnidarian Xlox are closer to the Central group. The evolutionary scenario of $X l o x$ 
thus remains unclear. Nevertheless, these results, coherent with our Hox analysis, suggest three possible models for the early evolution of Hox and ParaHox genes from an ancestral ProtoHox cluster.

\section{Results and Discussion}

HoxPred, an accurate tool to classify bilaterian Hox and ParaHox genes

We developed three new versions of HoxPred [36] to study the Hox and ParaHox genes at the scale of the metazoans (Table 1; see Materials and Methods for a description of these three versions). The "Bilateria" version aims at classifying Hox and ParaHox genes. The "Bilateria_relaxed" and "Vertebrate_relaxed" versions have been designed to study the evolutionary relationships between Hox and ParaHox genes. These versions were constructed and evaluated as in [35]. The prior probability for the control (CTL) group is very high in order to avoid misclassifications. HoxPred consequently shows tendency to classify divergent sequences into the CTL group, rather than any other group [35]. To assess the quality of the predictions, we applied the three new versions of HoxPred on a large set of 800 homeodomain sequences from 9 non-vertebrate species spanning various bilaterian phylogenetic groups, and which include well-characterized Hox and ParaHox genes (Additional file 2, Tables S1, S2, S3). Overall, the accuracy of all versions is high (Table 1). As expected, the "Bilateria" version provides an efficient and very stringent classification of Hox and ParaHox sequences (accuracy of 0.97): most Hox and ParaHox genes are correctly assigned to their class or group while non-Hox sequences are consistently classified in the control group. The two "relaxed" versions also provide correct predictions for Hox genes, with accuracy values higher than 0.90 .

To further investigate the evolution of Hox genes and the usefulness of HoxPred for its study, we applied the three new versions of HoxPred on a comprehensive dataset of 10,538 homeodomain sequences, from the UniprotKB database and 6 completely sequenced bilaterian genomes. To our knowledge, this is the first study on Hox sequences conducted on such a large scale. All results are freely available from the Datab'Hox database [36], through a friendly Web interface enabling complex queries and providing links to external databases. The HoxPred program is accessible on the same website [36]. Multiple alignments of Hox and ParaHox sequences ordered by PGs, are also available from this website and in the Additional file 3. Figure 2 and Additional file 2, Table S4 summarize the results obtained for the 6 species whose genome is completely sequenced: 3 lophotrochozoans (group including annelid worms and mollusks) (Capitella sp. I, Helobdella robusta and Lottia gigantea), 1 ecdysozoan (Daphnia pulex), and 2 deuterostomes (Strongylocentrotus purpuratus and Branchiostoma floridae). Results for Capitella sp. I and Branchiostoma floridae are coherent with published studies [42,43]. Of particular interest, we identified 11 Hox genes in the Lottia genome and found that these genes are clustered on a single scaffold and display the same orientation except for one of them, the last Posterior gene similar to Capitella Post1. Similarly, the 10 Daphnia genes are found on a single scaffold. A complex situation is found in Helobdella where 19 Hox genes are found on several different scaffolds. We also identified ParaHox genes (Figure 2 and Additional file 2, Table S4). While a single ParaHox gene $(C d x)$ is found in Daphnia, the 3 types of ParaHox genes are found in lophotrochozoans and deuterostomes (in agreement with previous studies $[16,19,42])$ and they are mostly organised into two- or three-genes clusters.

HoxPred therefore appears to be a suitable tool to identify Hox and ParaHox genes and we therefore used these identifications to address unsolved questions about the evolution of these genes.

\section{A global model for the evolution of Posterior Hox genes}

Posterior Hox genes of non-vertebrate bilaterians, including deuterostomes such as cephalochordates, urochordates, and ambulacraria (echinoderms and hemichordates), can not be confidently related to specific vertebrate PG using phylogenetic analyses $[21,29,43,44]$ (Additional file 1, Figure S2). It has been therefore

Table 1 Evaluation of predictions for the four versions of HoxPred.

\begin{tabular}{|c|c|c|c|c|c|}
\hline Reference & Version & Training Sequences & CTL group sequences & Homology groups & Accuracy \\
\hline$[35]$ & Vertebrate & Vertebrate & $\begin{array}{l}\text { RANDOM } \\
+ \\
\text { HOMEO }\end{array}$ & $\begin{array}{l}\text { PG1-14 } \\
+ \\
\text { orthologous groups }\end{array}$ & 0.97 \\
\hline new & Bilateria & Bilateria & $\begin{array}{l}\text { RANDOM } \\
+ \\
\text { HOMEO }\end{array}$ & $\begin{array}{l}\text { ANT/CENT/POST } \\
\text { GSX/XLOX/CDX }\end{array}$ & 0.97 \\
\hline new & Vertebrate_relaxed & Vertebrate & RANDOM & PG1-14 & 0.92 \\
\hline new & Bilateria_relaxed & Bilateria & RANDOM & ANT/CENT/POST & 0.98 \\
\hline
\end{tabular}

The Vertebrate version was not used in this study. 


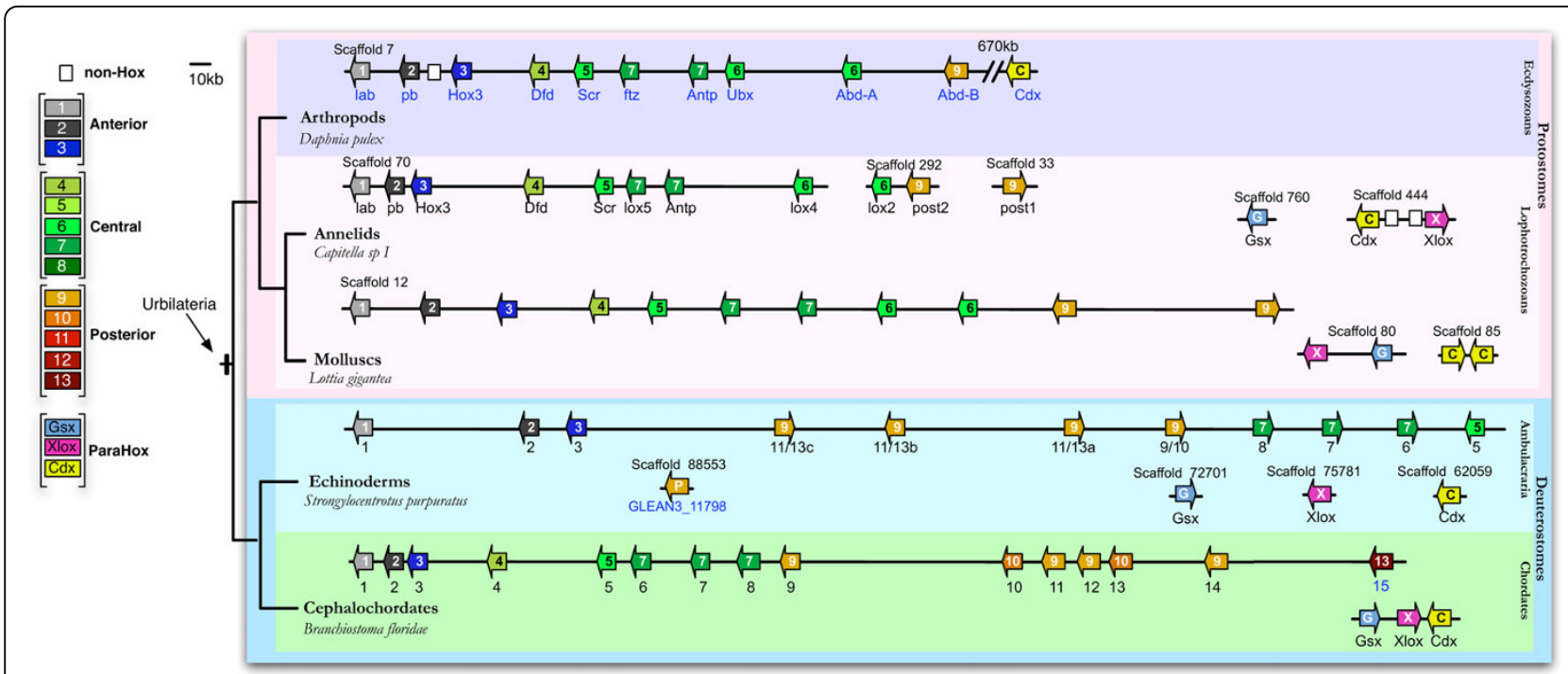

Figure 2 Genomic organization of the Hox genes identified with HoxPred in the genome-scale analyses. Hox and ParaHox genes are depicted with arrows indicating transcription orientation, over black lines representing the scaffolds. This representation takes into account the relative distance between the genes. The transcription orientation is the same as provided by the JGl genome browser. The color of the arrows relates to HoxPred classification (see color code on the left); white squares are non-Hox genes. The Hox cluster of Strongylocentrotus is from [46] and the ParaHox genes are from SpBase [63]. The Hox cluster of Branchiostoma is from [43], with the additional Branchiostoma Hox15 gene found in the genome assembly. Hellobdella genes are not indicated as they span many scaffolds, probably due to poor genome assembly. When available, gene names are specified: in black (from published studies $[16,42,43,46]$ ) or in blue (from the JGl genome browser or SpBase). An additional putative Hox gene, showing sequence similarities with $S p$-Hox $11 / 13 \mathrm{C}$, lies outside the Hox cluster in Strongylocentrotus. See Additional file 2, Table $\$ 4$ for the genomic coordinates.

proposed that the blurred relationships between Hox Posterior genes would be explained by an accelerated evolution rate of these genes, a process called 'Deuterostome Posterior Flexibility' [29]. An alternative hypothesis suggests multiple independent duplications to shape the posterior portion of the Hox clusters [29,45,46]. We used HoxPred to analyse the bilaterian Posterior Hox genes (Figures 2, 3A and Additional file 2, Table S4).

HoxPred assigned all amphioxus Posterior genes to PG9 and PG10, to the exception of Hox15, predicted as PG13 (Figure 3A). The latter has not been classified into any particular homology group with phylogenetic approaches, but it was clustered with PG13 with low statistical values [47]. Our own phylogenetic analyses show that the amphioxus Hox15 clustered with PG13 sequences, with a high posterior probability of 0.97 for the Bayesian tree (Additional file 1, Figure S2). Our data therefore suggest that the amphioxus Hox11-14 genes would have arisen from duplications of Hox9- and Hox10-like genes, independent to those which produced the vertebrate PG11 to PG14 Posterior Hox genes. HoxPred prediction for the Hox 14 gene thus brings additional support for an independent origin of the amphioxus and vertebrate Hox14 genes [47-50]. The assignment of Hox 15 to PG13 suggests that a PG13 gene was present in the chordate ancestor. The intriguing interspersed order of the PG9 and PG10 genes
(Figure 2) might be compatible with duplication of a Hox9-Hox10 genes segment. Comparison of surrounding non-coding sequence may give further insights into the duplication events.

In the case of the urochordate Oikopleura dioica, Hox 9 and Hox10 were classified into the PG9 and PG10, respectively, while both Hox 11 and Hox 12 were assigned to PG12 (Figure 3A; in agreement with the published phylogenetic tree [27]). These genes should be respectively renamed, e.g. Hox $12 a$ and Hox $12 b$. The ambulacraria posterior genes (9/10 and 11/13 groups) were all assigned to PG9. HoxPred also systematically classified ecdyosozoan Posterior Hox sequences in PG9. Finally, in lophotrochozans, the predictions were ambiguous: Post1 and Post 2 both alternated between PG9, PG12 and CTL, likely because these sequences are quite divergent [51]. An alternative hypothesis is that this uncertainty reflects an affinity for both PG9 and PG12, already present in an ancestral bilaterian $P G 9 / P G 12-l i k e$ posterior gene and retained in lophotrochozoans.

HoxPred predictions of posterior sequences have strong statistical support, with posterior probabilities similarly high as Anterior or Central sequences (Additional file 2, Tables S1, S4). However, as many of the predictions fall in the PG9 and PG10 groups, we conducted additional statistical tests (Additional file 1, Figure S3) to provide evidences that neither the PG9 nor 


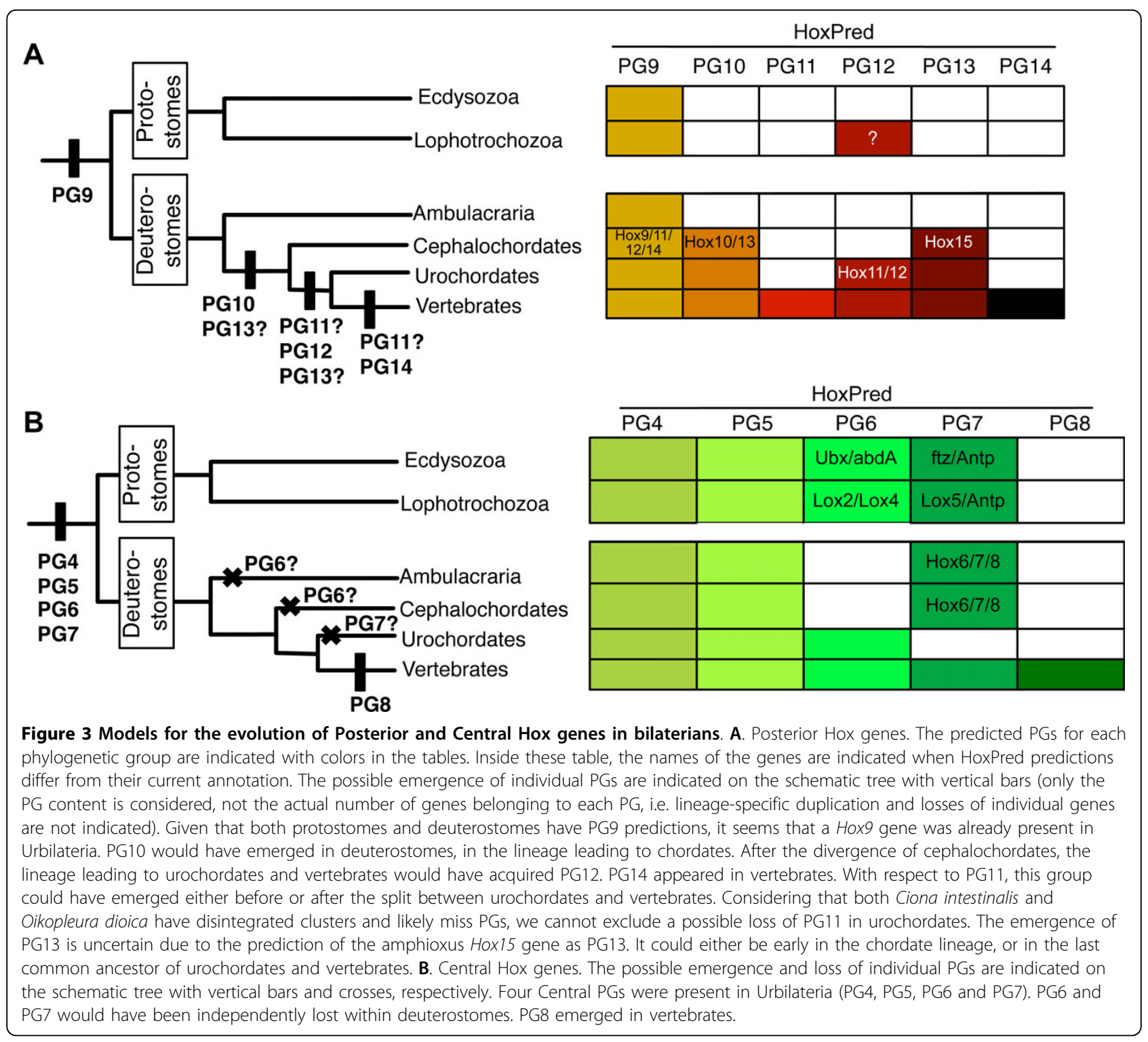

PG10 acts as a general 'Hox class' that would attract Hox sequences irrespective of their real identity.

Our analysis of HoxPred assignments favors the hypothesis of multiple independent duplications over the 'Deuterostome Posterior Flexibility' hypothesis alone, and allows to propose a global model for Posterior genes evolution in bilaterians (Figure 3A). In this model, we have only considered the PG9 predictions for protostomes, since the PG12 predictions in lophotrochozoans do not seem consistent. These predictions could be artifactual and might not indicate the presence of a PG12 gene in the protostome ancestor. Although poorly parsimonious in terms of duplication events, our model is supported by (i) the fact that urochordates, rather than cephalochordates, would be closer to vertebrates [52], thereby challenging the view that the amphioxus Hox cluster is the archetypal cluster from which aroused the vertebrate clusters - interestingly, there are more HoxPred predictions in common between vertebrates and urochordates than with cephalochordates; (ii) Many gene families, such as the bHLH family of transcription factors, have undergone multiple duplications specifically in the amphioxus [47,53]; (iii) Deuterostome Posterior Flexibity has been questioned in ambulacraria [44,45]; (iv) The amphioxus and vertebrate Hox 14 genes do not group together in phylogenetic trees [48].

\section{The bilaterian Central genes enigma}

While evolutionary relationships between Central genes from PG4 and PG5 across bilaterians were quite well resolved, phylogenetic approaches failed to decipher the relationships between the other three Central genes, 
eventually classified in a single broad PG6-8 group $[21,44,46]$. HoxPred predictions for PG4 and PG5 were consistent with tree-based annotation (Figure $3 \mathrm{~B}$ and Additional file 2, Table S4). For PG6-8, we found that ecdysozoan and lophotrochozoan genes are predicted into PG6 and PG7, with a strong tendency towards $U b x$, abd-A, Lox2 and Lox4 predicted as PG6, and ftz, Antp and Lox5 predicted as PG7 (Figure 3B and Additional file 2, Table S4). Ambulacraria and cephalochordates predictions are restricted to PG7, whereas urochordates only have PG6 predictions. Vertebrates is the only phylogenetic group having PG8 predictions, in addition to PG6 and PG7. These data suggest that PG4, PG5, PG6 and PG7 would have been present in the last common ancestor of all bilaterians (Urbilateria), and PG8 would have emerged in Vertebrates. The deuterostome predictions call for caution, as they would imply a loss of PG6 along with an expansion of PG7 genes in both Ambulacraria and cephalochordates, and a loss of PG7 in urochordates, a scenario which seems poorly parsimonious. However, urochordates have clearly lost members of many gene families $[53,54]$ and similar poorly parsimonious scenarios have been proposed for other genes families, for example the iroquois/Irx genes [55].

\section{Identification of Hox genes in Cnidaria}

Reconstructing the Hox repertoire of the Cnidaria/ Bilateria ancestor is a notorious challenge, as cnidarian 'Hox-like' genes are difficult to relate to the bilaterian homologous groups with traditionnal sequence similaritybased or phylogenetic analyses. The various phylogenetic studies published so far yielded a somewhat confuse picture $[12,27,31,32,56]$. We analysed the HoxPred assignments for all the homeobox genes from the fully sequenced Nematostella vectensis genome, as well as for 37 additional homeodomain sequences, from 11 other cnidarian species (Figure 4 and Additional file 2, Table S5). Using the "Bilateria" version of HoxPred, we found that most cnidarian Hox and ParaHox genes were classified as CTL, at the exception of a few Anterior and Gsx genes. This is not surprising given the divergent nature of the cnidarian Hox and ParaHox genes with respect to those from bilaterians. The "relaxed" versions of HoxPred, however, allow to classify cnidarian genes. The cnidarian Hox genes fall in Anterior, Central and Posterior groups predictions which strongly contradict the commonly accepted idea of a lack of Central Hox genes in Cnidaria [12,27], but see alternative hypotheses $[10,56]$. Genes predicted as Central include anthox 1 and anthox $1 a$ from the anthozoan Nematostella, which have been difficult to relate to a bilaterian group of homology, considered as either Central/Posterior [56], Posterior [12], cnidarianspecific [27] or even non-Hox [31]. Still, they are usually considered as non-anterior Hox genes $[12,27,31,56]$; their

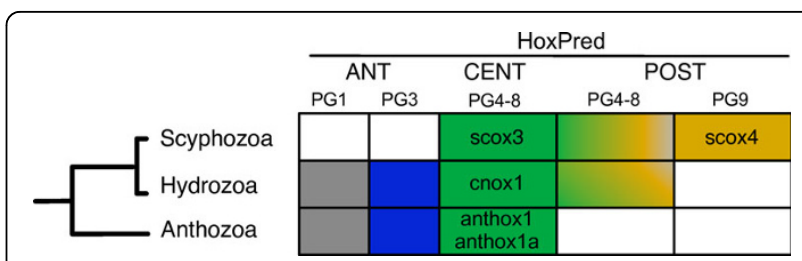

Figure 4 Summary of HoxPred predictions in cnidarians. The predicted homology group are indicated with colors in the table. ANT, CENT and POST predictions were obtained with the

"Bilateria_relaxed" version, while the PG predictions were obtained with the "Vertebrate_relaxed" version.

classification by HoxPred in the Central group is therefore in agreement with this view. Predictions as Central genes also encompass genes from hydrozoans (e.g. cnoxl from Eleutheria dichotoma) and a scyphozoan (scox3 from Cassiopea xamachana). Within the Anterior group, we found predictions for PG1 and PG3 that corroborate phylogenetic analyses $[18,27]$, but not for PG2 in contradiction to what has been reported in [12,32]. Predictions as Posterior genes, only found in hydrozoans and scyphozoans, are compatible with previous assignments [18,32]. However, most of the genes predicted as Posterior by the "Bilateria_relaxed" version of HoxPred are classified as Central genes with the "Vertebrate_relaxed" version, apart from scox4 that is predicted as Posterior by the two "relaxed" versions. This uncertainty may reflect that these genes may have arisen from an ancestral Central/Posterior gene.

In summary, our analysis indicates that cnidarians would possess three to four types of Hox genes, namely Anterior PG1, Anterior PG3, Central, and Posterior (or Central/Posterior) and therefore suggest that these three to four categories of Hox genes were already present in the last common ancestor of cnidarians and bilaterians (Figure 5).

\section{ParaHox predictions: implications for the ProtoHox models}

The ParaHox cluster of genes has long been supposed to be the sister cluster of the Hox cluster, with the Gsx, $X l o x$ and $C d x$ genes corresponding to the Anterior, PG3 and Posterior groups, respectively [13]. This view has been recently challenged by analyses of cnidaria data, by questioning both the cluster duplication model $[12,32]$ and the grouping of ParaHox genes with the Hox homology groups [18]. Assuming that Hox and ParaHox are nevertheless sister clusters which derived from a single ancestral ProtoHox cluster (reviewed in [18]), we tried to determine how the ParaHox genes can be related to the Hox genes. To do this we adopted a "Hox-centric" view, i.e. we used the "relaxed" versions of HoxPred to assign ParaHox genes in Hox PGs (Figure 5 and Additional file 2, Table S5, S6). Our attempt to perform the reverse analysis (i.e. to define in which 


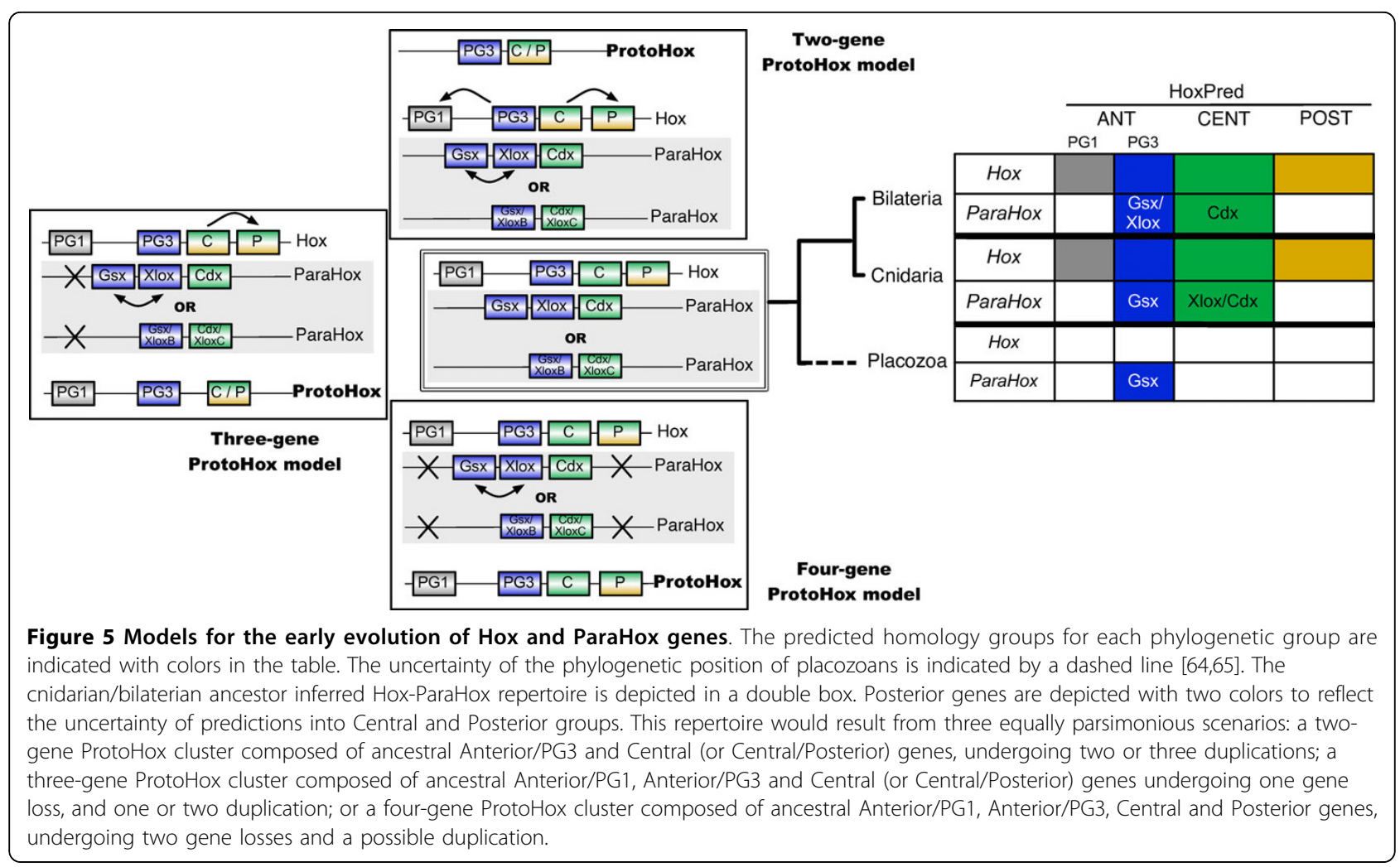

ParaHox groups individual Hox genes would be assigned) remained inconclusive due to the very small size of the training dataset.

We found that Gsx and Xlox genes were consistently predicted as Anterior/PG3 across bilaterians. Gs $x$ is similarly predicted as Anterior/PG3 in cnidarians and placozoans. The few Xlox genes from cnidarians predicted as Central could indicate that cnidarians Xlox would have emerged from a Central-like gene and are therefore distinct from the bilaterian PG3-derived Xlox genes. Alternatively, cnidarians Xlox might be related to bilaterian Xlox, but because of derived sequences as compared to their bilaterian counterpart, they may have been misclassified by HoxPred. Our results do not support the traditional grouping of Gsx with PG1, but are consistent with a recent phylogenetic analysis that regroups Gsx and Xlox into a PG2/PG3 group [18]. $C d x$ genes are consistently predicted in the Central group, rather than in the Posterior group.

Taken together with our data on cnidarian Hox genes, the cnidarian/bilaterian ancestor would have had a minimal Hox repertoire of four genes, composed of Anterior/ PG1, Anterior/PG3, Central and Posterior, and a minimal ParaHox repertoire of two genes $(G s x$ and $C d x$ ). Our results are coherent with three main ProtoHox models that seem equally parsimonious (Figure 5): (1) a fourgene ProtoHox cluster (Anterior/PG1, Anterior/PG3,
Central and Posterior) [13] - the four genes would have been conserved in the Hox cluster and two genes (Anterior/PG1 and Posterior) would have been lost in the ParaHox cluster; (2) a three-gene ProtoHox cluster (Anterior/PG1, Anterior/PG3 and Central/Posterior) previously proposed [10] but where the Central genes would have emerged specifically in bilaterians and not in cnidarians - one duplication would have produced the Posterior and Central Hox genes and one loss would explain the absence of Anterior/PG1 ParaHox genes; (3) a two-gene ProtoHox cluster (Anterior/PG3 and Central/ Posterior) - this is somewhat different to a previously suggested two-gene ProtoHox model (Anterior/PG1 and Posterior) [14] - in our model, the ancestral Anterior/ PG3 and Central/Posterior genes would have respectively given rise to the Hox genes of the PG3, PG1 and of the Central, Posterior groups, by two duplication events. In the three proposed models, Gsx and $C d x$ derive from ancestral Anterior/PG3-like and Central-like genes, respectively; Xlox might have evolved from a PG3-like gene by duplication prior to the cnidarians/bilaterians split, or independently in bilaterians (from PG3-like) and cnidarians (from Central-like).

\section{Conclusions}

The extensions of HoxPred presented here fulfill the needs for automatic Hox classification across all 
bilaterians. This method is well-suited for the evergrowing amount of sequences to analyse, by combining predictive accuracy and time efficiency (3000 sequences screened per hour on a standard laptop). HoxPred is easily accessible to the community through a userfriendly web page [36]. The HoxPred automatic classification for thousands of homeodomain sequences are provided in the freely available Datab'Hox database [36]. This new resource thereby offers supplementary information to the existing database HomeoDB [57], a manually curated database of homeobox genes with annotations based on published articles - when possible, links to HomeoDB are provided from Datab'Hox.

Our analyses illustrate the capacity of HoxPred to provide valuable predictions in ongoing genome projects. It is particularly appropriate for dispersed Hox clusters, as it directly pinpoints the potential Hox sequences. Beyond its classification purposes, we showed that HoxPred can also serve to study the evolution of Hox genes in metazoans. In this respect, we propose here evolutionary scenarios for several crucial questions. Bilaterian posterior Hox genes would have arisen from an ancestral PG9, with new homology groups arising in chordates, and in ambulacraria (group 11/13). Our model favours independent duplications, or a mixture of the two processes as suggested in [44,47], over the "Deuterostome Posterior Flexibility" hypothesis alone. It would be beneficial for the community to revise the nomenclature of the posterior Hox genes in non-vertebrate deuterostomes, so that the number of a gene with respect to its position within the cluster would not be confused with its homology group. Our evolutionary scenario for bilaterian Central genes suggests that Urbilateria would have possessed Central genes from PG4, PG5, PG6 and PG7; PG8 appearing later in vertebrates. Besides, our results bring additional support to the grouping of the Central protostome genes into Ubd-A-type and ftz/Antp/Lox5. We also provide further insights into the notoriously controversial relationships between cnidaria and bilateria Hox genes. Our analysis suggests that the cnidarian/bilaterian ancestor would have had a minimal Hox repertoire of four genes, from the Anterior/PG1, Anterior/PG3, Central and Posterior groups. HoxPred thus yields stimulating results in the context of the current views, by indicating the presence of cnidarian Central gene. Regarding the ParaHox genes, we suggest that Gsx derived from an ancestral PG3-like gene, while $C d x$ would be closer to the Central (Central/Posterior) genes, which is coherent with our data on cnidarian Hox genes. Xlox would have independently emerged from a PG3-like gene in bilaterians and from a Centrallike gene in cnidarians, or alternatively emerged earlier, from a PG3-like gene. Taken together, our results are consistent with three possible models for the early evolution of Hox and ParaHox genes: a two-gene (Anterior/PG3, Central/Posterior), a three-gene (Anterior/ PG1, Anterior/PG3 and Central/Posterior), or a fourgene (Anterior/PG1, Anterior/PG3, Central, Posterior) ProtoHox model.

\section{Methods}

\section{Sequence Datasets}

Sequences were mostly retrieved from UniprotKB [58] release 14.5. Strongylocentrotus purpuratus sequences were deduced from the annotation of the Hox cluster sequence (Genbank accession AC165428) and also retrieved from the sea urchin genome project http:// www.hgsc.bcm.tmc.edu/projects/seaurchin/[59]. The draft assemblies of the Capitella sp. I, Helobdella robusta, Lottia gigantea, Daphnia pulex and Branchiostoma floridae genomes are accessible at the DOE Joint Genome Institute http://www.jgi.doe.gov/. To analyse these genomes with HoxPred, the complete sets of homeodomain proteins were first filtered as in [35]. Here, only proteins matching the InterPro IPR001356 or IPR001827 homeobox domain were retrieved and further analysed.

\section{The four versions of HoxPred}

The initial HoxPred program [35] was constructed from vertebrate sequences only, and constitutes the "Vertebrate" version (not used in this study). The same procedure as in [35] was followed to produce the three new versions (including leave-one-out cross-validation, permutation tests and a variable selection step). The prior probabilities values were similar. For the "Bilateria" and "Bilateria_relaxed" versions, 6 new profiles corresponding to the Anterior, Central, Posterior, Gsx, Xlox and $\mathrm{Cdx}$ homology groups were developed. These profiles were constructed from alignments of 440 Hox and 37 ParaHox non-redundant homeodomain sequences (Additional file 4). These sequences were collected from publicly available databases and then manually curated. Sequences that were well-annotated and unambiguously classified into the Anterior, Central, Posterior, Gsx, Xlox and $\mathrm{Cdx}$ homology groups in a phylogenetic tree (not shown) were included in this training dataset. Contrary to the Vertebrate version, these sequences were extracted from various bilaterian phyla (including vertebrates). The "Vertebrate"/"Vertebrate_relaxed" and "Bilateria"/"Bilateria_relaxed" versions were respectively built upon the same collections of profiles. The main difference is related to the datasets used for the training of the discriminant analysis. In the "relaxed" versions, the control (CTL) group contains randomly-generated (RANDOM) sequences only. In the "non-relaxed" versions, the CTL group consists of both RANDOM and 
non-Hox homeodomain sequences (HOMEO) (1074 bilaterian sequences for the "Bilateria" version). The "non-relaxed" versions thus return all non-Hox sequences in the CTL group, while the "relaxed" versions can classify such sequences into Hox PG, thereby allowing the study of ParaHox sequences. All versions of HoxPred return classifications with posterior probabilities; the group with the highest posterior probability is considered as the prediction.

\section{Evaluation of HoxPred predictions}

The statistical evaluation of HoxPred predictions was performed on 800 public sequences $(89$ Hox and 711 non-Hox), with the programs compare-classes (option matrix file) and contigency-stats from the Regulatory Sequence Analysis Tools (RSAT) [60]/Network Analysis Tools (NeAT) [61], available at http://rsat.ulb.ac.be/rsat/ . The statistic used to evaluate the performance of HoxPred (Table 1), is the geometric accuracy, as previously described in [35]. For "relaxed" versions that are not intended for direct classification purposes, the accuracy is calculated for Hox genes only (excluding predictions of non-Hox homeobox genes), which underestimates the global accuracy.

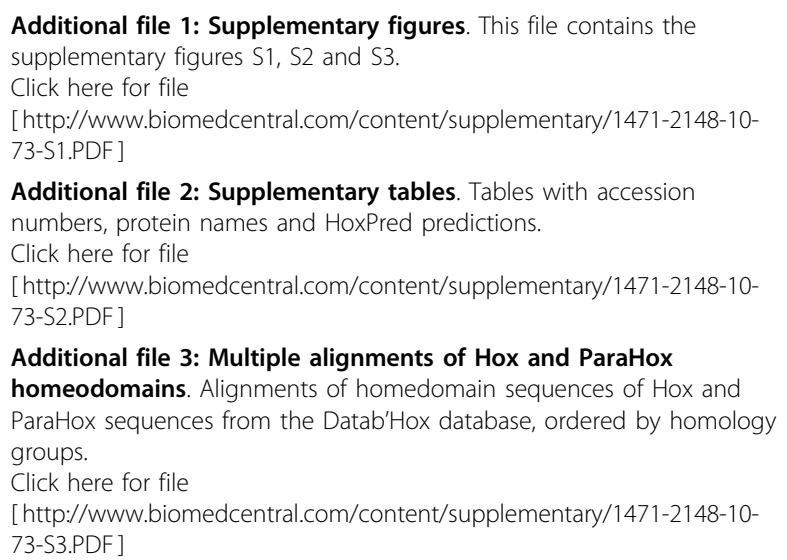

Additional file 2: Supplementary tables. Tables with accession numbers, protein names and HoxPred predictions.

Click here for file

[http://www.biomedcentral.com/content/supplementary/1471-2148-1073-S2.PDF ]

Additional file 3: Multiple alignments of Hox and ParaHox

homeodomains. Alignments of homedomain sequences of Hox and

ParaHox sequences from the Datab'Hox database, ordered by homology groups.

Click here for file

[http://www.biomedcentral.com/content/supplementary/1471-2148-1073-S3.PDF ]

Additional file 4: Accession numbers of training sequences. List of accession number and name of sequences used in the training dataset. Corresponding multiple alignments are available upon request.

Click here for file

[http://www.biomedcentral.com/content/supplementary/1471-2148-1073-S4.PDF ]

\footnotetext{
Acknowledgements

We are thankful to Jacques van Helden for precious support, Julia Lasserre for machine learning hints and David Ferrier for fruitful and encouraging discussions. This work was supported by the Vrije Universiteit Brussel [Geconcerteerde Onderzoeksactie 29], the ministère Français de la recherche (ANR non thématique), and the CNRS. BiGRe belongs to the Belgian Program on Interuniversity Attraction Poles, initiated by the Belgian Federal Science Policy Office, project P6/25 (BioMaGNet). We acknowledge the contribution of The US Department of Energy Joint Genome Institute in the production of the genomic sequences used in this study.
}

\section{Author details}

${ }^{1}$ Laboratoire de Bioinformatique des Génomes et des Réseaux (BiGRe), Université Libre de Bruxelles, Campus Plaine, CP 263, Boulevard du Triomphe, B-1050 Bruxelles, Belgium. '2Laboratory for Cell Genetics, Vrije Universiteit Brussel, Pleinlaan 2, B-1050 Brussels, Belgium. ${ }^{3}$ Belgian EMBnet Node, Université Libre de Bruxelles, CP 257, Bd du Triomphe, B-1050 Brussels, Belgium. ${ }^{4}$ Development and Neurobiology Program, Institut Jacques Monod, UMR 7592 CNRS/Université Paris Diderot - Paris 7, 15 rue Hélène Brion, 75205 Paris Cedex 13, France. ${ }^{5}$ UFR des Sciences du Vivant, Université Paris Diderot - Paris 7, 5, rue Marie-Andrée Lagroua Weill-Hallé, 75205 Paris Cedex 13, France. ${ }^{6}$ Current address: Department of Computational Molecular Biology, Max Planck Institute for Molecular Genetics, Ihnestrasse 73, 14195 Berlin, Germany.

\section{Authors' contributions}

MT-C extended HoxPred, performed the sequence analyses and designed the Datab'Hox database. MT-C and MV constructed the evolutionary scenarios, and MV performed the phylogenetic analyses. MV, VL and LL participated in the design and coordination of the study. MT-C drafted the manuscript and all the authors participated in the editing of the manuscript. All the authors read and approved the final manuscript.

Received: 11 October 2009 Accepted: 11 March 2010 Published: 11 March 2010

\section{References}

1. McGinnis W, Garber RL, Wirz J, Kuroiwa A, Gehring WJ: A homologous protein-coding sequence in Drosophila homeotic genes and its conservation in other metazoans. Cell 1984, 37(2):403-408.

2. Holland PW, Booth HA, Bruford EA: Classification and nomenclature of all human homeobox genes. BMC Biol 2007, 5:47.

3. McGinnis W, Krumlauf R: Homeobox genes and axial patterning. Cell 1992, 68(2):283-302.

4. Burke AC, Nelson CE, Morgan BA, Tabin C: Hox genes and the evolution of vertebrate axial morphology. Development 1995, 121(2):333-346.

5. Zakany J, Duboule D: The role of Hox genes during vertebrate limb development. Curr Opin Genet Dev 2007, 17(4):359-366.

6. Duboule $D$, Dollé $P$ : The structural and functional organization of the murine HOX gene family resembles that of Drosophila homeotic genes. EMBO J 1989, 8(5):1497-1505.

7. Dollé $P$, Izpisúa-Belmonte JC, Falkenstein $H$, Renucci A, Duboule D: Coordinate expression of the murine Hox-5 complex homoeoboxcontaining genes during limb pattern formation. Nature 1989, 342(6251):767-772.

8. Tschopp P, Tarchini B, Spitz F, Zakany J, Duboule D: Uncoupling time and space in the collinear regulation of Hox genes. PLoS Genet 2009, 5(3): e1000398.

9. Finnerty JR, Martindale MQ: The evolution of the Hox cluster: insights from outgroups. Curr Opin Genet Dev 1998, 8(6):681-687.

10. Ferrier DE, Holland PW: Ancient origin of the Hox gene cluster. Nat Rev Genet 2001, 2(1):33-38.

11. Garcia-Fernàndez J: The genesis and evolution of homeobox gene clusters. Nat Rev Genet 2005, 6(12):881-892.

12. Ryan JF, Mazza ME, Pang K, Matus DQ, Baxevanis AD, Martindale MQ, Finnerty JR: Pre-bilaterian origins of the Hox cluster and the Hox code: evidence from the sea anemone, Nematostella vectensis. PLOS ONE 2007, 2(1):e153.

13. Brooke NM, Garcia-Fernàndez J, Holland PW: The ParaHox gene cluster is an evolutionary sister of the Hox gene cluster. Nature 1998, 392(6679):920-922.

14. Garcia-Fernàndez J: Hox, ParaHox, ProtoHox: facts and guesses. Heredity 2005, 94(2):145-152.

15. Ferrier DE, Minguillón C: Evolution of the Hox/ParaHox gene clusters. Int J Dev Biol 2003, 47(7-8):605-611.

16. Osborne PW, Benoit G, Laudet V, Schubert M, Ferrier DEK: Differential regulation of ParaHox genes by retinoic acid in the invertebrate chordate amphioxus (Branchiostoma floridae). Dev Biol 2009, 327(1):252-262.

17. Hui JH, Holland PW, Ferrier DE: Do cnidarians have a ParaHox cluster? Analysis of synteny around a Nematostella homeobox gene cluster. Evol Dev 2008, 10(6):725-730. 
18. Quiquand M, Yanze N, Schmich J, Schmid V, Galliot B, Piraino S: More constraint on ParaHox than Hox gene families in early metazoan evolution. Dev Biol 2009, 328(2):173-187.

19. Hui J, Raible F, Korchagina N, Dray N, Samain S, Magdelenat G, Jubin C, Segurens B, Balavoine G, Arendt D, et al: Features of the ancestral bilaterian inferred from Platynereis dumerilii ParaHox genes. BMC Biol 2009, 7(1):43.

20. Holland PW, Garcia-Fernàndez J: Hox genes and chordate evolution. Dev Biol 1996, 173(2):382-395

21. Balavoine $G$, de Rosa R, Adoutte A: Hox clusters and bilaterian phylogeny. Mol Phylogenet Evol 2002, 24(3):366-373.

22. Ferrier DE: Hox genes: Did the vertebrate ancestor have a Hox14? Curr Biol 2004, 14(5):R210-211.

23. Long S, Byrne M: Evolution of the echinoderm Hox gene cluster. Evol Dev 2001, 3(5):302-311.

24. Duboule D: The rise and fall of Hox gene clusters. Development 2007, 134(14):2549-2560.

25. Finnerty JR, Martindale MQ: Ancient origins of axial patterning genes: Hox genes and ParaHox genes in the Cnidaria. Evol Dev 1999, 1(1):16-23.

26. Banerjee-Basu S, Baxevanis AD: Molecular evolution of the homeodomain family of transcription factors. Nucleic Acids Res 2001, 29(15):3258-3269.

27. Chourrout D, Delsuc F, Chourrout P, Edvardsen RB, Rentzsch F, Renfer E, Jensen MF, Zhu B, de Jong P, Steele RE, et al: Minimal ProtoHox cluster inferred from bilaterian and cnidarian Hox complements. Nature 2006 442(7103):684-687.

28. Baurain D, Brinkmann $H$, Philippe $H$ : Lack of resolution in the animal phylogeny: closely spaced cladogeneses or undetected systematic errors? Mol Biol Evol 2007, 24(1):6-9.

29. Ferrier DE, Minguillón C, Holland PW, Garcia-Fernàndez J: The amphioxus Hox cluster: deuterostome posterior flexibility and Hox14. Evol Dev 2000, 2(5):284-293.

30. Kourakis MJ, Martindale MQ: Combined-method phylogenetic analysis of Hox and ParaHox genes of the metazoa. J Exp Zool 2000, 288(2):175-191.

31. Kamm K, Schierwater B, Jakob W, Dellaporta SL, Miller DJ: Axial patterning and diversification in the cnidaria predate the Hox system. Curr Biol 2006, 16(9):920-926.

32. Chiori $R$, Jager $M$, Denker E, Wincker $P$, Da Silva C, Le Guyader $H$, Manuel M, Quéinnec E: Are Hox genes ancestrally involved in axial patterning? Evidence from the hydrozoan Clytia hemisphaerica (Cnidaria). PLOS ONE 2009, 4(1):e4231.

33. Sharkey M, Graba Y, Scott MP: Hox genes in evolution: protein surfaces and paralog groups. Trends Genet 1997, 13(4):145-151.

34. Telford MJ: Turning Hox "signatures" into synapomorphies. Evol Dev 2000 2(6):360-364

35. Thomas-Chollier M, Leyns L, Ledent V: HoxPred: automated classification of Hox proteins using combinations of generalised profiles. BMC Bioinformatics 2007, 8:247.

36. HoxPred/Datab'Hox. http://cege.vub.ac.be/hoxpred/.

37. Fonseca NA, Vieira CP, Holland P, Vieira J: Protein evolution of ANTP and PRD homeobox genes. BMC Evol Biol 2008, 8(1):200.

38. Bucher P, Karplus K, Moeri N, Hofmann K: A flexible motif search technique based on generalized profiles. Comput Chem 1996, 20(1):3-23.

39. Thomas-Chollier M, Ledent V: Comparative phylogenomic analyses of teleost fish Hox gene clusters: lessons from the cichlid fish Astatotilapia burtoni: comment. BMC Genomics 2008, 9(1):35.

40. Amemiya CT, Wagner GP: Animal evolution: when did the 'Hox system' arise? Curr Biol 2006, 16(14):R546-548.

41. Lanfear R, Bromham $L$ : Statistical tests between competing hypotheses of Hox cluster evolution. Syst Biol 2008, 57(5):708-718.

42. Fröbius $A C$, Matus $D Q$, Seaver $E C$ : Genomic organization and expression demonstrate spatial and temporal Hox gene colinearity in the lophotrochozoan Capitella sp. I. PLOS ONE 2008, 3(12):e4004

43. Amemiya CT, Prohaska SJ, Hill-Force A, Cook A, Wasserscheid J, Ferrier DE, Pascual-Anaya J, Garcia-Fernàndez J, Dewar K, Stadler PF: The amphioxus Hox cluster: characterization, comparative genomics, and evolution. J Exp Zool B Mol Dev Evol 2008.

44. Monteiro AS, Ferrier DE: Hox genes are not always Colinear. Int J Biol Sci 2006, 2(3):95-103.

45. Peterson KJ: Isolation of Hox and Parahox genes in the hemichordate Ptychodera flava and the evolution of deuterostome Hox genes. $\mathrm{Mol}$ Phylogenet Evol 2004, 31(3):1208-1215.
46. Cameron RA, Rowen L, Nesbitt R, Bloom S, Rast JP, Berney K, ArenasMena C, Martinez P, Lucas S, Richardson PM, et al: Unusual gene order and organization of the sea urchin hox cluster. J Exp Zool B Mol Dev Evol 2006, 306(1):45-58.

47. Holland L, Albalat R, Azumi K, Benito-Gutierrez E, Blow M, Bronner-Fraser M, Brunet F, Butts T, Candiani S, Dishaw L, et al: The amphioxus genome illuminates vertebrate origins and cephalochordate biology. Genome Res 2008, 18(7):1100-1111.

48. Powers TP, Amemiya CT: Evidence for a Hox14 paralog group in vertebrates. Curr Biol 2004, 14(5):R183-184.

49. Kuraku S, Takio Y, Tamura K, Aono H, Meyer A, Kuratani S: Noncanonical role of Hox14 revealed by its expression patterns in lamprey and shark. Proc Natl Acad Sci USA 2008.

50. Kuraku S, Meyer A: The evolution and maintenance of Hox gene clusters in vertebrates and the teleost-specific genome duplication. Int J Dev Biol 2009, 53(5-6):765-773.

51. de Rosa R, Grenier JK, Andreeva T, Cook CE, Adoutte A, Akam M, Carroll SB, Balavoine G: Hox genes in brachiopods and priapulids and protostome evolution. Nature 1999, 399(6738):772-776.

52. Delsuc F, Brinkmann $H$, Chourrout D, Philippe $H$ : Tunicates and not cephalochordates are the closest living relatives of vertebrates. Nature 2006, 439(7079):965-968.

53. Simionato $E$, Ledent $V$, Richards $G$, Thomas-Chollier $M$, Kerner $P$, Coornaert D, Degnan BM, Vervoort M: Origin and diversification of the basic helix-loop-helix gene family in metazoans: insights from comparative genomics. BMC Evol Biol 2007, 7:33.

54. Edvardsen RB, Seo HC, Jensen MF, Mialon A, Mikhaleva J, Bjordal M, Cartry J, Reinhardt R, Weissenbach J, Wincker P, et al: Remodelling of the homeobox gene complement in the tunicate Oikopleura dioica. Curr Biol 2005, 15(1):R12-13.

55. Kerner $P, I \mathrm{kmi} A$, Coen D, Vervoort M: Evolutionary history of the iroquois/ Irx genes in metazoans. BMC Evol Biol 2009, 9:74.

56. Ryan JF, Burton PM, Mazza ME, Kwong GK, Mullikin JC, Finnerty JR: The cnidarian-bilaterian ancestor possessed at least 56 homeoboxes: evidence from the starlet sea anemone, Nematostella vectensis. Genome Biol 2006, 7(7):R64

57. Zhong Y-F, Butts T, Holland PWH: HomeoDB: a database of homeobox gene diversity. Evol Dev 2008, 10(5):516-518.

58. UniProt-Consortium: The universal protein resource (UniProt). Nucleic Acids Res 2008, , 36 Database: D190-195.

59. Sea Urchin Genome Sequencing Consortium, Sodergren E, Weinstock GM, Davidson EH, Cameron RA, Gibbs RA, Angerer RC, Angerer LM, Arnone MI, Burgess DR, et al: The genome of the sea urchin Strongylocentrotus purpuratus. Science 2006, 314(5801):941-952.

60. Thomas-Chollier M, Sand O, Turatsinze JV, Janky R, Defrance M, Vervisch E, Brohée S, van Helden J: RSAT: regulatory sequence analysis tools. Nucleic Acids Research 2008.

61. Brohée S, Faust K, Lima-Mendez G, Sand O, Janky R, Vanderstocken G, Deville $Y$, van Helden J: NeAT: a toolbox for the analysis of biological networks, clusters, classes and pathways. Nucleic Acids Research 2008, , 36 Web Server: W444-451.

62. Sigrist CJA, Cerutti L, Hulo N, Gattiker A, Falquet L, Pagni M, Bairoch A Bucher P: PROSITE: a documented database using patterns and profiles as motif descriptors. Brief Bioinformatics 2002, 3(3):265-274.

63. Cameron RA, Samanta M, Yuan A, He D, Davidson E: SpBase: the sea urchin genome database and web site. Nucleic Acids Research 2009, , 37 Database: D750-754

64. Schierwater B, Eitel M, Jakob W, Osigus HJ, Hadrys H, Dellaporta SL, Kolokotronis SO, DeSalle R: Concatenated analysis sheds light on early metazoan evolution and fuels a modern "urmetazoon" hypothesis. PLoS Biol 2009, 7(1):e20

65. Philippe H, Derelle R, Lopez P, Pick K, Borchiellini C, Boury-Esnault N, Vacelet J, Renard E, Houliston E, Quéinnec E, et al: Phylogenomics revives traditional views on deep animal relationships. Curr Biol 2009, 19(8):706-712.

doi:10.1186/1471-2148-10-73

Cite this article as: Thomas-Chollier et al:: A non-tree-based comprehensive study of metazoan Hox and ParaHox genes prompts new insights into their origin and evolution. BMC Evolutionary Biology 2010 10:73 